\title{
Ionic diffusion through confined geometries: from Langevin equations to partial differential equations
}

\author{
Boaz Nadler $^{1}$, Zeev Schuss ${ }^{2}$, Amit Singer ${ }^{2}$ and R S Eisenberg ${ }^{3}$ \\ ${ }^{1}$ Department of Mathematics, Yale University, New-Haven, CT 06520, USA \\ 2 Department of Applied Mathematics, Tel-Aviv University, Ramat-Aviv 69978, Tel-Aviv, Israel \\ ${ }^{3}$ Department of Molecular Biophysics and Physiology, Rush Medical Center, 1750 Harrison \\ Street, Chicago, IL 60612, USA
}

Received 3 October 2003

Published 21 May 2004

Online at stacks.iop.org/JPhysCM/16/S2153

DOI: $10.1088 / 0953-8984 / 16 / 22 / 015$

\begin{abstract}
Ionic diffusion through and near small domains is of considerable importance in molecular biophysics in applications such as permeation through protein channels and diffusion near the charged active sites of macromolecules. The motion of the ions in these settings depends on the specific nanoscale geometry and charge distribution in and near the domain, so standard continuum type approaches have obvious limitations. The standard machinery of equilibrium statistical mechanics includes microscopic details, but is also not applicable, because these systems are usually not in equilibrium due to concentration gradients and to the presence of an external applied potential, which drive a non-vanishing stationary current through the system. We present a stochastic molecular model for the diffusive motion of interacting particles in an external field of force and a derivation of effective partial differential equations and their boundary conditions that describe the stationary non-equilibrium system. The interactions can include electrostatic, Lennard-Jones and other pairwise forces. The analysis yields a new type of Poisson-Nernst-Planck equations, that involves conditional and unconditional charge densities and potentials. The conditional charge densities are the non-equilibrium analogues of the well studied pair correlation functions of equilibrium statistical physics. Our proposed theory is an extension of equilibrium statistical mechanics of simple fluids to stationary non-equilibrium problems. The proposed system of equations differs from the standard Poisson-Nernst-Planck system in two important aspects. First, the force term depends on conditional densities and thus on the finite size of ions, and second, it contains the dielectric boundary force on a discrete ion near dielectric interfaces. Recently, various authors have shown that both of these terms are important for diffusion through confined geometries in the context of ion channels.
\end{abstract}




\section{Introduction}

With ongoing advances in technology and experimental techniques, the physical systems that are either studied or designed become smaller and smaller, nowadays reaching nanometre and nanosecond length and timescales, respectively. In this paper we focus on a particular type of system, containing a nanoscale (nearly picoscale) pore connected to two large reservoirs of electrolyte solutions. Two examples of such a system, of great importance to molecular biophysics and biotechnological applications, are ionic permeation through protein channels [1,2] and through carbon nanotubes [3].

These nanoscale systems exhibit a range of phenomena not encountered in larger macroscopic systems. For example, due to the confined geometry of the pore through which the fluid flows, ions typically cannot pass by each other, leading to single filing phenomena, complex relations between unidirectional currents and other nonlinear phenomena in mixtures [2]. The confined geometry enhances the importance of individual ion-ion interactions, which are thought to be the cause of the high selectivity of these nanopores to specific ionic species [4].

The function of these systems depends not only on the nanoscale atomic details and geometry of the pore, but also on experimentally controlled macroscopic variables, such as the applied electrostatic potential and the surrounding bath concentrations. Therefore, the function of these systems involves many different time and length scales, from the femtosecond and ångström scales for the motion of single water molecules, up to the microsecond timescale at which current measurements are made, and micro- to millimetre distances at which measuring devices are placed. One final important feature worth mentioning is that these systems almost always function away from equilibrium, as does almost all biology. These nanopores separate regions of different concentrations and a voltage difference is usually present across them. Therefore, these systems can be viewed as nanodevices, with well defined input and output relations that are the purpose of the device.

Many of these biological systems are of interest because they are highly sensitive, with atomic scale details controlling macroscopic flows. Mutations or modifications to the atomic structure of the nanopore lead to significant changes in its characteristics. Thus, the theoretical analysis of such systems necessarily resides at the interface between continuum and discrete atomic molecular physics, presenting new challenges in non-equilibrium statistical physics. On the one hand, due to the importance of confined geometry, discrete ion-ion interactions and non-uniform dielectric coefficient, standard continuum equations of electrolyte solutions, such as Poisson-Boltzmann (PB), Poisson-Fokker-Planck, or Poisson-Nernst-Planck (PNP) are not valid [5-7]. On the other hand, due to the wide range of time and length scales involved-mixing nanoscale and continuum - direct molecular dynamics (MD) simulations are impractical for the study of the macroscopic function of these systems. Regretfully, even coarse grained Brownian dynamics (BD) simulations are not always the appropriate tool to study such systems. For example, it is not possible to study the effects of a $10 \mu \mathrm{M}$ concentration of calcium ions in a $100 \mathrm{mM} \mathrm{Na}^{+} \mathrm{Cl}^{-}$electrolyte in a simulation that contains only a few hundred ions, even though the biological effects of such trace concentrations are often of overwhelming importance [8]. Therefore, there is a need for a hierarchy of models each valid in its own scale, and connections between them.

While the standard PNP system may not be valid in confined geometries, it is important to note the advantages of a continuum description of nanoscale systems. The computational complexity of continuum descriptions, that typically involve the solution of a system of coupled partial differential equations, is often orders of magnitude lower than that of corresponding $\mathrm{BD}$ or MD simulations. Another significant advantage of continuum descriptions over BD 
and MD simulations is that they easily accommodate non-equilibrium boundary conditions for the macroscopic electrostatic potential and ionic concentrations. Simulations in the chemical tradition have difficulty with such conditions (see however [9]). The goal, then, is to derive continuum equations for non-equilibrium systems that include molecular details, absent in the PNP theory. We note that for the corresponding equilibrium problem, the theory of equilibrium statistical mechanics of simple fluids incorporates particle-particle interactions by the Bogolyubov-Born-Green-Kirkwood-Yvon (BBGKY) hierarchy [10, 11]. This hierarchy, coupled with a closure relation, has proven successful in the calculation of a number of important macroscopic properties of electrolytes from their underlying molecular interactions [12].

The main problem discussed in this paper is thus how to generalize the theory of equilibrium statistical mechanics of simple fluids, which is based on the equilibrium Boltzmann distribution, to stationary non-equilibrium problems. To this end, we note that one of the key missing elements in the equilibrium theory is the dynamics of the moving particles. The Boltzmann distribution defines the probability of configurations but does not include a description of how individual particles move in the system. In non-equilibrium problems there is usually a net flow of particles. It is not possible to compute net fluxes from equilibrium theories. In this paper, following our previous work [5], we present a molecular model of permeation, based on diffusive motion of ions, and propose a mathematical averaging procedure that results in a hierarchy of Poisson and Nernst-Planck type equations containing conditional and unconditional charge densities. The proposed conditional system, called C-PNP, includes molecular details such as excluded volume effects and the dielectric force on a discrete ion that are absent in the standard PNP system. Recently, various authors have shown that both of these terms are important for diffusion through confined geometries, in the context of ion channels [4, 13-16]. The C-PNP system, along with a closure relation and boundary conditions, provides a theoretical framework for the study of non-equilibrium diffusing systems. The C-PNP system reduces to the BBGKY hierarchy when equilibrium boundary conditions are given, thus generalizing equilibrium statistical mechanics to stationary non-equilibrium problems. Application of C-PNP to ion channels can be expected to predict blocking and possibly selectivity.

The paper is organized as follows. In section 2 we describe the standard PoissonBoltzmann and Poisson-Nernst-Planck theories and discuss their limitations in confined geometries. Then, in section 3 , we briefly review the theory of equilibrium statistical mechanics of simple fluids, and its limited applicability to non-equilibrium systems. The main results of this paper are described in sections 4 and 5, where we formulate a non-equilibrium stochastic molecular model based on trajectories, and present a mathematical derivation of the corresponding continuum equations. We conclude in section 6 with summary and discussion.

\section{Standard continuum treatments and their failure in confined systems}

We consider the setup shown in figure 1 (left). A rigid nanopore connects two reservoirs of electrolyte solutions. The system is kept in a stationary non-equilibrium condition by an external feedback mechanism that maintains average concentrations $c_{\mathrm{L}}$ and $c_{\mathrm{R}}$ in the left and right reservoirs, respectively, and an average applied voltage $V$ across them. The problem at hand is to compute the stationary ionic current through the nanopore as a function of the parameters of the system: the geometry, dielectric coefficients, and fixed charge distribution of the pore, the atomic characteristics of the ions (e.g., radii, ion-ion interaction forces, and their diffusion coefficients), and as a function of the experimentally controlled macroscopic variables, $c_{\mathrm{L}}, c_{\mathrm{R}}$ and $V$. 


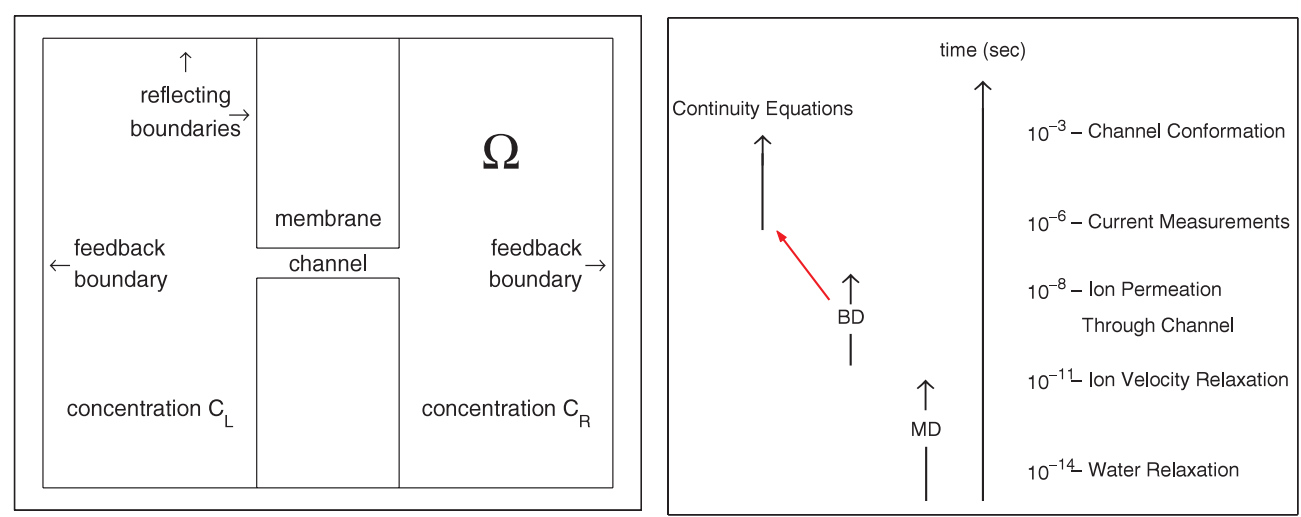

Figure 1. A typical system with a nanoscale channel pore connecting two large electrolyte solutions (left), and typical timescales of various processes for permeation through a protein channel (right).

(This figure is in colour only in the electronic version)

Throughout this paper we consider modelling approaches at the level of the primitive model $[10,19]$ that do not consider explicitly the individual molecules of the solvent (water), but rather treat them as a continuous structure-less dielectric, and as a viscous and noisy medium. Some of the limitations of this approach are discussed in the summary section. For simplicity, we consider an ionic solution with only two ionic species, with positive and negative charges, such as $\mathrm{Na}^{+} \mathrm{Cl}^{-}$, although this assumption can easily be relaxed.

Two of the most common mean-field continuum theories of ionic solutions are the equilibrium Poisson-Boltzmann (PB) and the non-equilibrium Poisson-Nernst-Planck (PNP) theories $[10,11]$. Both PB and PNP assume a constitutive relation of the form

$$
J_{\mathrm{p}}(\boldsymbol{x})=-D_{\mathrm{p}}\left(\nabla c_{\mathrm{p}}(\boldsymbol{x})+c_{\mathrm{p}}(\boldsymbol{x}) \frac{e z_{\mathrm{p}}}{k_{\mathrm{B}} T} \nabla \psi(\boldsymbol{x})\right)
$$

between the local averaged particle flux of the positive ionic species at location $x$, called $\boldsymbol{J}_{\mathrm{p}}(\boldsymbol{x})$, their concentration $c_{\mathrm{p}}(\boldsymbol{x})$, and a potential of mean field $\psi(\boldsymbol{x})$. In this equation $k_{\mathrm{B}}$ is Boltzmann's constant, $T$ is temperature, $D_{\mathrm{p}}$ is the diffusion coefficient of the positive ions and $z_{\mathrm{p}}$ is their valence. A similar equation for the flux of the negative ions is also assumed. The equilibrium PB theory assumes that the local flux vanishes everywhere,

$$
\boldsymbol{J}_{\mathrm{p}}(\boldsymbol{x})=\boldsymbol{J}_{\mathrm{n}}(\boldsymbol{x})=0,
$$

while the PNP theory assumes conservation of flux, e.g. the Nernst-Planck equation,

$$
\nabla \cdot \boldsymbol{J}_{\mathrm{p}}(\boldsymbol{x})=\nabla \cdot \boldsymbol{J}_{\mathrm{n}}(\boldsymbol{x})=0 .
$$

The simplicity of these two theories lies in their crude approximation for the potential of the mean field. Both PB and PNP assume that the potential $\psi(x)$ satisfies Poisson's equation with the average concentrations $c_{\mathrm{p}}(\boldsymbol{x})$ and $c_{\mathrm{n}}(\boldsymbol{x})$,

$$
\nabla \cdot[\varepsilon(\boldsymbol{x}) \nabla \psi(\boldsymbol{x})]=-e\left[z_{\mathrm{p}} c_{\mathrm{p}}(\boldsymbol{x})-z_{\mathrm{n}} c_{\mathrm{n}}(\boldsymbol{x})+\rho_{\text {fixed }}(\boldsymbol{x})\right],
$$

where $\varepsilon(\boldsymbol{x})$ is the location dependent dielectric coefficient and $\rho_{\text {fixed }}$ is the concentration of fixed charges in the system. In this approximation, the electrostatic field exerted on an ion at location $\boldsymbol{x}$ is computed by the gradient of the mean electrostatic potential (3).

The PB theory is often the starting point of modern electrochemistry, with the famous Debye-Hückel theory of ionic shielding $[11,17]$. The PNP theory has also found numerous 
applications in the study of electrolyte transport, plasma physics and in modelling of semiconductor devices $[11,18]$.

Both of these theories represent the ions as a continuous charge distribution in an ambient mean potential field, defined by the Poisson equation (3). Both theories neglect effects due to the finite size of ions and due to the discrete nature of their charge. The limitations of the PB approach, even in bulk electrolyte solutions with moderate to high concentrations, are well known $[11,17,19]$. Recently, the failure of PB and PNP in confined geometries has also been shown both in simulations $[6,7]$ and in theoretical treatments [5].

\section{Equilibrium statistical mechanics of simple fluids}

The preceding discussion indicates that the problem at hand is how to include molecular details in non-equilibrium continuum type equations. It is important to note that molecular details can be included in continuum models with satisfactory results. For example, the theory of equilibrium statistical mechanics of simple fluids yields continuum type equations with molecular detail, and has proven successful in the computation of many macroscopic quantities $[11,12]$, some of which (e.g., activity of ions) are difficult to determine in MD or BD simulations. We now briefly describe the key elements in equilibrium theory and its limitations for non-equilibrium systems. For simplicity we consider the canonical (NVT) ensemble formulation.

The classical description of the statistical physics of interacting particles (at the level of the primitive model) starts with a finite system of $N$ particles in a finite volume $V$ at a fixed temperature $T$ with $N$ particles at locations $\boldsymbol{x}_{1}, \ldots, \boldsymbol{x}_{N}$. It is usually assumed that the potential of the forces acting on the ions, $U\left(x_{1}, x_{2}, \ldots, x_{N}\right)$, is explicitly known, and is a sum of pair radial interactions,

$$
U\left(x_{1}, x_{2}, \ldots, x_{N}\right)=\sum_{i<j} U_{i, j}\left(\left|x_{i}-x_{j}\right|\right),
$$

with the role of forces at the boundary and electrostatic interactions with boundary charges often being ignored. The configurational partition function for this system is defined as

$$
Q_{N}(\beta)=\int \cdots \int_{V^{N}} \mathrm{e}^{-\beta U\left(\boldsymbol{x}_{1}, \ldots, \boldsymbol{x}_{N}\right)} \prod_{i=1}^{N} \mathrm{~d} \boldsymbol{x}_{i},
$$

where $\beta=1 / k_{\mathrm{B}} T$. The main assumption of the theory is that the probability of a given configuration of the $N$ particles follows the Boltzmann distribution,

$$
p\left(\boldsymbol{x}_{1}, \boldsymbol{x}_{2}, \ldots, \boldsymbol{x}_{N}\right)=\frac{\mathrm{e}^{-\beta U\left(\boldsymbol{x}_{1}, \ldots, \boldsymbol{x}_{N}\right)}}{Q_{N}} .
$$

Since the number of particles in a given system is usually of the order of Avogadro's number, one must consider a reduced description of the system; thus sometimes the marginal densities of only one or two particles are considered. By definition, the average physical density at location $x_{1}$ is given by

$$
\rho\left(\boldsymbol{x}_{1}\right)=N \int \cdots \int_{V^{N-1}} p\left(\boldsymbol{x}_{1}, \ldots, \boldsymbol{x}_{N}\right) \prod_{i=2}^{N} \mathrm{~d} \boldsymbol{x}_{i} .
$$

In equilibrium statistical mechanics, it is often customary to consider the limit of very large systems, namely $N,|V| \rightarrow \infty$, such that $N /|V|=\rho$. In this limit, it is possible to show that $\rho(\boldsymbol{x})$ satisfies the following equation, known as the first BBGKY equation,

$$
\nabla \rho(\boldsymbol{x})+\rho(\boldsymbol{x}) \int \frac{1}{k_{\mathrm{B}} T} \nabla_{x} U_{1,2}(\boldsymbol{x}-\boldsymbol{y}) \rho(\boldsymbol{y} \mid \boldsymbol{x}) \mathrm{d} \boldsymbol{y}=0,
$$


where $\rho(\boldsymbol{y} \mid \boldsymbol{x})$ describes the conditional density of particles at $\boldsymbol{y}$, given the presence of a particle at $\boldsymbol{x}$. By definition,

$$
\rho(\boldsymbol{y} \mid \boldsymbol{x})=\frac{\rho(\boldsymbol{x}, \boldsymbol{y})}{\rho(\boldsymbol{x})}
$$

where $\rho(\boldsymbol{x}, \boldsymbol{y})$ is the joint physical concentration of a pair of particles.

For homogeneous infinite systems $\rho(\boldsymbol{x})$ is uniform, due to symmetry, and thus equal to the bulk concentration $\rho$. The microscopic structure of the solution is described by the pair correlation function $g_{2}(\boldsymbol{x}, \boldsymbol{y})$, which is the non-dimensional version of $\rho(\boldsymbol{x}, \boldsymbol{y})$, given by

$g_{2}\left(\boldsymbol{x}_{1}, \boldsymbol{x}_{2}\right)=\frac{\rho\left(\boldsymbol{x}_{1}, \boldsymbol{x}_{2}\right)}{\rho^{2}}=\lim _{N, V \rightarrow \infty} N(N-1) \int \cdots \int_{V^{N-2}} p\left(\boldsymbol{x}_{1}, \boldsymbol{x}_{2}, \ldots, \boldsymbol{x}_{N}\right) \prod_{i=3}^{N} \mathrm{~d} \boldsymbol{x}_{i}$.

The pair correlation function is important for the determination of many thermodynamic properties of an equilibrium system, such as the free energy, pressure, chemical potential and compressibility of electrolytes, to name just a few [11, 12].

By differentiating (7) with respect to $x_{1}$, it is possible to show that $g_{2}$ satisfies the second BBGKY equation, which depends on the higher order triplet correlation function $g_{3}$,

$k_{\mathrm{B}} T \nabla_{x_{1}} g_{2}+g_{2} \nabla_{x_{1}} U_{1,2}+\rho \int \nabla_{x_{1}} U_{1,3}\left(\left|\boldsymbol{x}_{1}-\boldsymbol{x}_{3}\right|\right) g_{3}\left(\boldsymbol{x}_{1}, \boldsymbol{x}_{2}, \boldsymbol{x}_{3}\right) \mathrm{d} \boldsymbol{x}_{3}=0$.

Equations (6) and (8) correspond to a system with only one species of interacting particle. In the case of two species of interacting particle-say positive and negative ionsthere are two singlet density functions, $g_{1}^{\mathrm{p}}$ and $g_{1}^{\mathrm{n}}$, for the positive and negative species, respectively. There are also four pair correlation functions, $g_{2}^{\alpha, \beta}$, where $\alpha, \beta$ are one of the four possible combinations $(\alpha, \beta) \in\{(\mathrm{p}, \mathrm{p}),(\mathrm{p}, \mathrm{n}),(\mathrm{n}, \mathrm{p}),(\mathrm{n}, \mathrm{n})\}$, though obviously $g_{2}^{\mathrm{p}, \mathrm{n}}(\boldsymbol{x}, \boldsymbol{y})=g_{2}^{\mathrm{n}, \mathrm{p}}(\boldsymbol{y}, \boldsymbol{x})$. All of these quantities satisfy first and second BBGKY equations similar to (6) and (8).

The BBGKY equations constitute an infinite hierarchy of forwardly coupled integrodifferential equations. Further approximations are needed to solve the problem. For example, approximate solutions for the single and pair densities are often computed using closure relations, such as HNC or MSA, relating higher order correlation functions to lower order correlation functions, and the relevant thermodynamical quantities are calculated from the resulting densities $[11,12]$.

\subsection{Equilibrium versus non-equilibrium statistical mechanics}

In contrast to the simple equilibrium system described in the previous section, the biophysical systems we consider are in steady state, but not equilibrium. Our system is connected to an energy source, either a natural one, as in living organisms, or an artificial one that mimics the natural one, but also allows experimental control of parameters. Usually the nanopore carries a steady net flux of particles (e.g., either into or out of a biological cell). A steady state system carrying a constant flow cannot be described by the equilibrium theory, since the Boltzmann distribution assumes a symmetrical velocity distribution with zero mean and thus zero net flux. The question, then, is how to generalize the equilibrium theory to such non-equilibrium systems. In the next section we construct a non-equilibrium analogue of the Boltzmann distribution and use it to calculate fluxes.

It is instructive to note that the first BBGKY equation (6) can be rewritten as

$$
\boldsymbol{J}(\boldsymbol{x})=0,
$$


where $\boldsymbol{J}(\boldsymbol{x})$ is the average flux at location $\boldsymbol{x}$,

$$
\boldsymbol{J}(\boldsymbol{x})=-D\left[\nabla \rho(\boldsymbol{x})-\rho(\boldsymbol{x}) \frac{\overline{\boldsymbol{f}}(\boldsymbol{x})}{k_{\mathrm{B}} T}\right],
$$

with $D$ the diffusion coefficient and $\bar{f}(x)$ the average force on a particle at $\boldsymbol{x}$, given by

$$
\overline{\boldsymbol{f}}(\boldsymbol{x})=-\int \nabla_{x} U_{1,2}(\boldsymbol{x}-\boldsymbol{y}) \rho(\boldsymbol{y} \mid \boldsymbol{x}) \mathrm{d} \boldsymbol{y} .
$$

Upon comparison of equation (9) with (1) and (2) for the PB and PNP fluxes, it is tempting to write the following equation for the non-equilibrium case,

$$
\nabla \cdot \boldsymbol{J}(\boldsymbol{x})=0 .
$$

This result, however plausible, cannot be derived from the assumptions of equilibrium statistical mechanics, because those assumptions imply $\boldsymbol{J}(\boldsymbol{x})=0$. We present below a derivation of this non-equilibrium equation from the dynamics of diffusing ions.

\section{Non-equilibrium statistical mechanics}

\subsection{A trajectory based approach}

Our point of departure for the statistical description of non-equilibrium systems of diffusing particles is a probability measure defined by their trajectories in phase space, rather than by the statistics of points in configuration space, which is the starting point of traditional equilibrium statistical mechanics.

First, we introduce some notation. We consider a finite domain $\Omega$, that consists of the two macroscopic reservoirs and the connecting rigid nanopore (see figure 1, left). Its boundary $\partial \Omega$ is composed of reflecting boundaries $\partial \Omega_{\mathrm{R}}$ and the feedback boundaries $\partial \Omega_{\mathrm{F}}$. We assume that there are $N^{h}$ ions of species $h\left(h=\mathrm{Ca}^{2+}, \mathrm{Na}^{+}, \mathrm{Cl}^{-}, \ldots\right)$ in $\Omega$, which are numbered at time $t=0, \sum_{h} N^{h}=N$, and we follow their trajectories at all times $t>0$. The coordinates of a point are $\boldsymbol{x}=(x, y, z)$, while the location and velocity coordinates of the $j$ th ion of species $h$ at time $t$ are $\boldsymbol{x}_{j}^{h}(t)$ and $\boldsymbol{v}_{j}^{h}(t)$, respectively. According to our assumptions, an ion that reaches $\partial \Omega_{\mathrm{F}}$ is instantly re-injected by the feedback mechanism at one or another part of the boundary, so that its individual identity is preserved, and consequently the total number of ions inside $\Omega$ is fixed at all times. The feedback mechanism serves as the energy source for the system, keeping it in stationary non-equilibrium by keeping average concentrations $c_{\mathrm{L}}$ and $c_{\mathrm{R}}$ in the left and right reservoirs and a constant applied voltage $V$ across the system. In experimental situations, electronic devices and chemical apparatus provide the energy and feedback. In biological systems, metabolic machinery and the active transport systems it fuels provide the energy and feedback.

The evolution of the joint probability density function (PDF) of all the ions and water molecules in this system can be described by the Liouville equation. However, at the level of the primitive model, we follow the evolution of the PDF of only the ions in the system, which is a lower dimensional projection of the former.

Since the motion of ions in solution is strongly overdamped, on timescales larger than the relaxation time of the solution, memory effects due to the thermal motion of the solvent can be neglected [10], and the joint motion of only the ions can be described as diffusion with interactions. Therefore, our starting point is a memoryless system of coupled Langevin equations for the different ion species $h=\mathrm{Ca}^{2+}, \mathrm{Na}^{+}, \mathrm{Cl}^{-}$, etc, $j=1, \ldots, N^{h}$,

$$
\ddot{\boldsymbol{x}}_{j}^{h}+\gamma^{h}\left(\boldsymbol{x}_{j}^{h}\right) \dot{\boldsymbol{x}}_{j}^{h}=\frac{\boldsymbol{f}_{j}^{h}}{M^{h}}+\sqrt{2 \varepsilon^{h} \gamma^{h}\left(\boldsymbol{x}_{j}^{h}\right)} \dot{\boldsymbol{w}}_{j}^{h},
$$


where a dot on a variable indicates differentiation with respect to time, $\gamma^{h}\left(x^{h}\right)$ is the location dependent friction coefficient per unit mass, $M^{h}$ is the effective mass of an ion of species $h$ and $\varepsilon^{h}=k_{\mathrm{B}} T / M^{h}$. The force on the $j$ th ion of species $h$ is $f_{j}^{h}$ and includes all ion-ion interactions. It thus depends on the locations of all ions. The functions $\dot{\boldsymbol{w}}_{j}^{h}$ are, by assumption, independent standard Gaussian white noises. Thus, the effects of the solvent are modelled as a source of noise and friction for ionic motion and as an averaged dielectric coefficient for the ion-ion interactions. We discuss the limitations of these approximations in section 6.

\subsection{The Fokker-Planck equation}

We define by $p_{N}\left(\boldsymbol{x}_{1}, \ldots, \boldsymbol{x}_{N}, \boldsymbol{v}_{1}, \ldots, \boldsymbol{v}_{N}\right)$ the stationary PDF of the system of all $N$ ions. Since the coupled motion of all ions is governed by the Langevin system (10) with independent noise terms, the stationary PDF $p_{N}$ satisfies the multi-dimensional stationary Fokker-Planck equation (FPE) [20]:

$$
0=\sum_{h} \sum_{j=1}^{N^{h}} \mathcal{L}_{j}^{h} p_{N},
$$

where $\mathcal{L}_{j}^{h}$ is the Fokker-Planck operator acting on the phase space coordinates of the $j$ th ion of species $h$. It is given by

$$
\mathcal{L}_{j}^{h} p_{N}=\nabla_{\boldsymbol{v}_{j}^{h}} \cdot\left(\gamma^{h}\left(\boldsymbol{x}_{j}^{h}\right) \boldsymbol{v}_{j}^{h}-\frac{\boldsymbol{f}_{j}^{h}}{M^{h}}\right) p_{N}+\Delta_{\boldsymbol{v}_{j}^{h}} \varepsilon^{h} \gamma^{h}\left(\boldsymbol{x}_{j}^{h}\right) p_{N}-\boldsymbol{v}_{j}^{h} \cdot \nabla_{x_{j}^{h}} p_{N},
$$

where the operators $\nabla_{v}$ and $\Delta_{v}$ denote the gradient and the Laplacian with respect to the variable $\boldsymbol{v}$, respectively. Equation (11) is defined in the $3 N$ dimensional region $\left(\boldsymbol{x}_{1}, \ldots, \boldsymbol{x}_{N}\right) \in \Omega^{N}$ and $\left(\boldsymbol{v}_{1}, \ldots, \boldsymbol{v}_{N}\right) \in \boldsymbol{R}^{3 N}$.

The solution $p_{N}$ of the stationary FPE (11) is the non-equilibrium analogue of the Boltzmann distribution. It is the stationary transition probability density function of the $6 \mathrm{~N}$ dimensional trajectory of the system in phase space and thus it is also the probability density function of the particle configurations in phase space. Obviously, since the FPE is defined in a finite region, a unique solution is determined only after specification of appropriate boundary conditions. As shown in [21], if no-flux boundary conditions are given, the FPE (11) can be solved explicitly and the solution is the Boltzmann distribution. Thus, our formulation of a non-equilibrium system reduces to the equilibrium theory in this special case. It is thus clear that the boundary conditions are what drive the system out of equilibrium. The boundary conditions for the FPE (11) need to be determined from the action of the feedback mechanism at the boundaries and will be described in a separate publication [21].

Finally, we note that a time dependent FPE, similar to (11), was suggested [10, 22] as the starting point for the study of the transport characteristics of bulk electrolytes, by an analysis of the decay into equilibrium of transient infinite non-equilibrium electrolyte systems.

\section{The C-PNP system}

With the interpretation of the stationary joint transition PDF of the phase-space trajectories, $p_{N}\left(x_{1}, \ldots, v_{1}, \ldots\right)$, as the probability density of configurations of all particles in phase space, we can follow the steps taken in the theory of equilibrium statistical mechanics, and study the single and pair densities. An equation similar to (5), for $c^{h}(x)$, the time-averaged steady state physical concentration of ions of species $h$ at location $x_{1}^{h}$, is given by

$$
c^{h}\left(\boldsymbol{x}_{1}^{h}\right)=N^{h} \int_{\Omega^{N-1} \times \mathcal{R}^{3 N}} p_{N}\left(\boldsymbol{x}_{1}^{h}, \ldots, \boldsymbol{v}_{1}^{h}, \ldots\right) \prod_{\left(i, h^{\prime}\right) \neq(1, h)} \mathrm{d} \boldsymbol{x}_{i}^{h^{\prime}} \prod_{i, h^{\prime}} \mathrm{d} \boldsymbol{v}_{i}^{h^{\prime}} .
$$


In the equilibrium case, equation (4) gives an explicit expression for $p_{N}$, so that some of the integrations in (12) can be performed explicitly. Moreover, in deriving the BBGKY equation, the limit $N,|V| \rightarrow \infty$ is taken. In the non-equilibrium case, the system remains finite and $p_{N}$ is not known explicitly. What is known is only that $p_{N}$ satisfies the FPE equation (11) in a finite domain. However, by integration of this equation over all particle coordinates but one, the following Nernst-Planck type equation for the concentration $c^{h}(\boldsymbol{x})$ can be derived [5]:

$$
0=-\nabla_{x} \cdot J^{h}(x),
$$

where $\boldsymbol{J}^{h}(\boldsymbol{x})$ is the flux density of type $h$ ions, given by

$$
\boldsymbol{J}^{h}(\boldsymbol{x})=-D^{h}(\boldsymbol{x})\left[\nabla c^{h}(\boldsymbol{x})-\frac{\overline{\boldsymbol{f}}^{h}(\boldsymbol{x})}{k_{\mathrm{B}} T} c^{h}(\boldsymbol{x})\right],
$$

where $D^{h}(\boldsymbol{x})=k_{\mathrm{B}} T / M^{h} \gamma^{h}(\boldsymbol{x})$ is the local diffusion coefficient of species $h$. The quantity $\overline{\boldsymbol{f}}^{h}(\boldsymbol{x})$ in (14) is the average force on a single ion of type $h$. It is given by

$$
\bar{f}^{h}(\boldsymbol{x})=\int_{\Omega^{N-1}} \boldsymbol{f}_{1}^{h} p_{N-1}\left(\tilde{\boldsymbol{x}}_{1}^{h} \mid \boldsymbol{x}_{1}^{h}=\boldsymbol{x}\right) \mathrm{d} \tilde{\boldsymbol{x}}_{1}^{h},
$$

where $\tilde{\boldsymbol{x}}_{1}^{h}$ is the vector of all $N-1$ particle coordinates except $\boldsymbol{x}_{1}^{h}$ and $p_{N-1}\left(\tilde{\boldsymbol{x}}_{1}^{h} \mid \boldsymbol{x}_{1}^{h}=\boldsymbol{x}\right)$ is the conditional probability density of the $N-1$ remaining ions given that the first ion of species $h$ is located at $\boldsymbol{x}$.

In the case of charged ions in solution, the ion-ion interaction forces are pair-wise additive, and thus the force on the first ion of species $h$ can be written as

$$
\boldsymbol{f}_{1}^{h}=\boldsymbol{f}_{e d}^{h}\left(\boldsymbol{x}_{1}^{h}\right)+\sum_{\left(i, h^{\prime}\right) \neq(1, h)} \boldsymbol{f}^{h, h^{\prime}}\left(\boldsymbol{x}_{i}^{h^{\prime}}, \boldsymbol{x}_{1}^{h}\right),
$$

where $f^{h, h^{\prime}}$ is the ion-ion interaction force that an ion of type $h^{\prime}$ acts on an ion of type $h$. It includes both Coulombic interactions as well as short range interactions, such as excluded volume or Lennard-Jones forces. The force $f_{e d}^{h}$ contains both the effects of an applied external field as well as the dielectric self-force near dielectric boundaries [5, 13, 23]. Interactions between charges in the system and boundary charges, including both fixed and induced charges, may determine the entire behaviour of the system, e.g., to stop the flow of ions through an open pore. These interactions change the energy of the system also in the equilibrium case.

As shown in [5], with the specific form (16) for the force on a single ion, equation (15) for the average force simplifies to

$$
\overline{\boldsymbol{f}}^{h}(\boldsymbol{x})=\boldsymbol{f}_{e d}^{h}(\boldsymbol{x})+\overline{\boldsymbol{f}}_{\mathrm{SR}}^{h}(\boldsymbol{x})-\left.z^{h} e \nabla_{y} \bar{\phi}^{h}(\boldsymbol{y} \mid \boldsymbol{x})\right|_{y=\boldsymbol{x}},
$$

where

$$
\overline{\boldsymbol{f}}_{\mathrm{SR}}^{h}(\boldsymbol{x})=\sum_{h^{\prime}} \int_{\Omega} \boldsymbol{f}_{\mathrm{SR}}^{h, h^{\prime}}(\boldsymbol{y}, \boldsymbol{x}) c^{h^{\prime} \mid h}(\boldsymbol{y} \mid \boldsymbol{x}) \mathrm{d} \boldsymbol{y}
$$

is the average short range force on a type $h$ ion, $z^{h}$ is the valence of type $h$ ions and $\bar{\phi}^{h}(\boldsymbol{y} \mid \boldsymbol{x})$ is the conditional electrostatic potential at $\boldsymbol{y}$ given a type $h$ ion at $\boldsymbol{x}$. It satisfies the (conditional) Poisson equation

$$
\nabla_{\boldsymbol{y}} \cdot\left[\varepsilon(\boldsymbol{y}) \nabla_{\boldsymbol{y}} \bar{\phi}^{h}(\boldsymbol{y} \mid \boldsymbol{x})\right]=-e \sum_{h^{\prime}} z^{h^{\prime}} c^{h^{\prime} \mid h}(\boldsymbol{y} \mid \boldsymbol{x}),
$$

where $\varepsilon(y)$ is the dielectric coefficient at $\boldsymbol{y}$. In both equation (17) and (18), $c^{h^{\prime} \mid h}(\boldsymbol{y} \mid x)$ is the conditional concentration of $h^{\prime}$ ions at $\boldsymbol{y}$ given an $h$-type ion at $\boldsymbol{x}$. In terms of unconditional quantities, it is given by

$$
c^{h^{\prime} \mid h}(\boldsymbol{y} \mid \boldsymbol{x})=\frac{c^{h, h^{\prime}}(\boldsymbol{x}, \boldsymbol{y})}{c^{h}(\boldsymbol{x})} .
$$


To summarize, the density $c^{h}(\boldsymbol{x})$ satisfies a Nernst-Planck type equation (13), with an average force $\bar{f}^{h}$ that is the sum of a dielectric self force, an averaged short range force (17) and an averaged electrostatic force. The latter is a solution of a conditional Poisson equation that depends on conditional densities, in contrast to the unconditional densities in the standard PNP formulation, equation (3). We call this resulting system of conditional Poisson and Nernst-Planck equations C-PNP.

The NP equation (13) is defined in the finite domain $\Omega$. Therefore, in addition to the determination of the averaged force $\bar{f}^{h}$, boundary conditions on $\partial \Omega$ must be specified in order to determine the unique solution for $c^{h}(\boldsymbol{x})$. Obviously, on $\partial \Omega_{\mathrm{R}}, c^{h}(\boldsymbol{x})$ satisfies no flux boundary conditions,

$$
\left.\boldsymbol{J}^{h}(\boldsymbol{x}) \cdot \boldsymbol{\nu}\right|_{x \in \partial \Omega_{\mathrm{R}}}=0 .
$$

In addition, on $\partial \Omega_{\mathrm{F}}$, according to our assumptions, the average concentrations $c^{h}(x)$ are maintained at fixed known values $c_{\mathrm{F}}^{h}(\boldsymbol{x})$ by the feedback mechanism. Therefore, regardless of the exact method by which the feedback mechanism maintains these average concentrations,

$$
c^{h}(\boldsymbol{x})=c_{\mathrm{F}}^{h}(\boldsymbol{x}), \quad \text { for } \boldsymbol{x} \in \partial \Omega_{\mathrm{F}} .
$$

\subsection{The C-PNP hierarchy}

An important difference between the PNP and the C-PNP systems is that the C-PNP system is not closed, because, as seen from (17) and (18), the averaged force in the Nernst-Planck equation (13) depends on conditional higher order concentrations. Specifically, we consider the equation for the pair concentrations. Employing similar methods to those of [5], we obtain that $c^{h, h^{\prime}}(\boldsymbol{x}, \boldsymbol{y})$ satisfies the six-dimensional Nernst-Planck equation

$$
\nabla_{x^{h}} \cdot \boldsymbol{J}_{\boldsymbol{x}^{h}}^{h, h^{\prime}}(\boldsymbol{x}, \boldsymbol{y})+\nabla_{\boldsymbol{y}^{h^{\prime}}} \cdot \boldsymbol{J}_{\boldsymbol{y}^{h^{\prime}}}^{h, h^{\prime}}(\boldsymbol{x}, \boldsymbol{y})=0,
$$

where

$$
\boldsymbol{J}_{x^{h}}^{h, h^{\prime}}(\boldsymbol{x}, \boldsymbol{y})=-D^{h}(\boldsymbol{x})\left[\nabla_{x} c^{h, h^{\prime}}(\boldsymbol{x}, \boldsymbol{y})-\frac{\overline{\boldsymbol{f}}^{h, h^{\prime}}(\boldsymbol{x}, \boldsymbol{y})}{k_{\mathrm{B}} T} c^{h, h^{\prime}}(\boldsymbol{x}, \boldsymbol{y})\right]
$$

and $\overline{\boldsymbol{f}}^{h, h^{\prime}}(\boldsymbol{x}, \boldsymbol{y})$ is the average force on an ion of species $h$ located at $\boldsymbol{x}$, given an ion of species $h^{\prime}$ located at $\boldsymbol{y}$. The second flux $\boldsymbol{J}_{y^{h^{\prime}}}^{h, h^{\prime}}$ is given by a similar expression. For the case of pairwise additive forces, this force can be simplified to

$$
\overline{\boldsymbol{f}}^{h, h^{\prime}}(\boldsymbol{x}, \boldsymbol{y})=\boldsymbol{f}_{e d}^{h}(\boldsymbol{x})+\boldsymbol{f}^{h, h^{\prime}}(\boldsymbol{x}, \boldsymbol{y})+\overline{\boldsymbol{f}}_{\mathrm{SR}}^{h, h^{\prime}}(\boldsymbol{x}, \boldsymbol{y})-\left.e z^{h} \nabla_{z} \bar{\phi}^{h, h^{\prime}}(\boldsymbol{z} \mid \boldsymbol{x}, \boldsymbol{y})\right|_{z=x}
$$

where $\bar{f}_{\mathrm{SR}}^{h, h^{\prime}}$ and $\bar{\phi}^{h, h^{\prime}}$ are the higher order analogues of (17) and (18), which depend on the third order conditional concentrations $c^{h^{\prime \prime} \mid h, h^{\prime}}$. Equation (19) is the non-equilibrium analogue of the second BBGKY equation (8), and as in equilibrium, determination of the forces $\overline{\boldsymbol{f}}^{h, h^{\prime}}(\boldsymbol{x}, \boldsymbol{y})$ requires knowledge of the triplet densities $c^{h, h^{\prime}, h^{\prime \prime}}(\boldsymbol{x}, \boldsymbol{y}, \boldsymbol{z})$.

Similarly, it is possible to write an equation for the triplet density, whose average forces depend on fourth order conditional densities. We arrive this way at an infinite hierarchy of conditional Poisson and Nernst-Planck type equations, all defined in finite domains. The resulting equations are very similar to those used in the study of macroscopic bulk dynamical properties of electrolytes [10], where the time dependence of similar equations is considered in infinite domains. In these studies, closure relations between the triplet and pair densities, similar to those of equilibrium statistical mechanics, are employed in order to compute the average forces [24]. Closure relations require further physics or approximations, or both.

In our case, however, since we are concerned with a finite system in non-equilibrium, a closure relation between the triplet and the pair densities is not enough to close the 
system. Specifically, the Smoluchowski type equation (19) is defined in the finite domain $(\boldsymbol{x}, \boldsymbol{y}) \in \Omega \times \Omega$. Therefore, to uniquely determine its solution, boundary conditions have to be prescribed on the domain boundaries, e.g. for $(\boldsymbol{x}, \boldsymbol{y}) \in \partial \Omega \times \Omega$ and $(\boldsymbol{x}, \boldsymbol{y}) \in \Omega \times \partial \Omega$. Only after these boundary conditions are specified, the pair concentration $c^{h, h^{\prime}}(\boldsymbol{x}, \boldsymbol{y})$ is completely determined, provided the forces $\overline{\boldsymbol{f}}^{h, h^{\prime}}(\boldsymbol{x}, \boldsymbol{y})$ are known. As in the case of the single ion densities, the boundary conditions for the pair densities should also be determined by the action of the feedback mechanism. The derivation of boundary conditions for the pair concentrations, as well as for higher order densities, requires a more detailed description of the feedback mechanism [21].

\subsection{PNP revisited}

The simplest possible closure is

$$
c^{h^{\prime} \mid h}(\boldsymbol{y} \mid \boldsymbol{x})=c^{h^{\prime}}(\boldsymbol{y}),
$$

which assumes independence of ions and thus neglects ion-ion finite size effects. Therefore, it is also necessary to neglect all short range forces in this approximation, if one wishes to be consistent. This closure recovers the (unconditional) PNP system, but with an additional force term, $\boldsymbol{f}_{e d}$, the dielectric boundary force on a single ion near dielectric interfaces. This term represents the forces on a single ion by surface charges induced by the ion itself at dielectric interfaces $[13,23]$. This force term has a crucial importance in the determination of the permeation characteristics of the gramicidin channel, see [13].

The above analysis of this closure clarifies the assumptions and validity of PB and PNP. These two theories are obtained as approximations of the exact BBGKY and C-PNP systems by use of the simple closure (20) that neglects both the discreteness of charge and the finite size of ions. Therefore, in any system where discreteness of charge and the finite size of particles are important, such as near dielectric interfaces or in confined regions, the validity of these theories is questionable.

\section{Summary and discussion}

The function of biological systems such as ion channels typically involves the atomic control of macroscopic flows. These channels are nanoscale non-equilibrium systems connecting large electrolyte reservoirs, so their description involves many different length and timescales. Yet, the majority of published work on channels typically considers only a single level of modelling, which is valid or computable on limited time and length scales. In order to study the function of such systems, connections between theories at different levels are essential. In this paper we presented a connection between the level of Brownian dynamics (BD), represented by the system of coupled Langevin equations (10) and of continuum theories, represented by the C-PNP hierarchy (13), (19) and so forth. This connection is shown graphically as the diagonal arrow in figure 1 (right).

Since Brownian dynamics simulations are based on the numerical solution of the Langevin system (10), our analysis shows an equivalence between BD simulations and the C-PNP hierarchy. This equivalence can be used to validate specific closure relations as well as $\mathrm{BD}$ computer codes by comparing the results of the two computations. The resulting CPNP hierarchy is not closed as it involves conditional and unconditional charge densities. We find that it is the boundary conditions that drive the system out of equilibrium. When equilibrium boundary conditions are imposed the equilibrium BBGKY hierarchy is recovered from the C-PNP equations. While the single ion densities satisfy simple concentration and no 
flux boundary conditions, the corresponding conditions for the higher order densities are not obvious and require further analysis [21].

The two main force terms that appear in the C-PNP system and are absent in standard PNP are the dielectric boundary force and the effects due to the finite size of ions. Both of these terms have been shown to be important in the context of narrow protein channels [4, 13-16]. However, to the best of our knowledge, the validity of various closure relations has not yet been considered in confined and highly non-homogeneous systems.

The derivation presented in this paper is at the level of the primitive model and thus relies on a few assumptions concerning the properties of the implicit solvent. One of these assumptions is that the noise terms of different ions are independent. This assumption may not hold in very narrow regions occupied by one or more ions and only a few water molecules, where ions and water may move in a highly coordinated fashion. The analysis of this configuration requires separate analysis. Another assumption concerns the representation of the solvent as an effective dielectric constant. Given the substantial frequency dependence [25], and possible location dependence of induced charge, higher resolution methods must be used, along with experimentation, to evaluate this representation and its parameters. Specifically, the description of induced charge by a dielectric coefficient (with its inherent assumption of a linear invariant relation between induced charge and local electrical field) must be validated, and the frequency/time and location dependence of this dielectric coefficient evaluated. Such high resolution calculations are not trivial because they themselves must be shown to represent the electric field accurately over the relevant length and timescales. We note that in the context of proteins, the dielectric coefficient for dielectric boundary forces may not be the same as the coefficient for charge-charge interactions [26].

Finally, we note that in this paper we assumed that the nanopore is rigid. If this is not the case, then the dynamics of the fluctuations of the nanopore structure from its average configuration should be coupled to the Langevin equations for the motion of all the ions as described in [5]. This would result in a conditional Poisson equation with a conditional averaged structure of the nanopore, that depends on the location of the mobile ion inside it. It seems likely that gating can be described by such a coupled system of equations.

\section{References}

[1] Eisenberg R S 1999 From structure to function in open ionic channels J. Membr. Biol. 171 1-24

[2] Hille B 2001 Ionic Channels of Excitable Membranes 3rd edn (Massachusetts: Sinauer Associates)

[3] Joseph S, Mashl R J, Jacobsson E and Aluru N R 2003 Ion channel based biosensors: ionic transport in carbon nanotubes Technical Proc. 2003 Nanotechnology Conf. vol 1, pp 158-61

[4] Gillespie D, Nonner W and Eisenberg R S 2002 Coupling Poisson-Nernst-Planck and density functional theory to calculate ion flux J. Phys.: Condens. Matter 14 12129-45

[5] Schuss Z, Nadler B and Eisenberg R S 2001 Derivation of PNP equations in bath and channel from a molecular model Phys. Rev. E 64036116

[6] Moy G, Corry B, Kuyucak S and Chung S 2000 Tests of continuum theories as models of ion channels. I. Poisson-Boltzmann theory versus Brownian dynamics Biophys. J. 78 2349-63

[7] Corry B, Kuyucak S and Chung S 2000 Tests of continuum theories as models of ion channels. II. PoissonNernst-Planck theory versus Brownian dynamics Biophys. J. 78 2364-81

[8] Alberts B, Johnson A, Lewis J, Raff M, Roberts K and Walter P 2002 Molecular Biology of the Cell 4th edn (New York: Garland)

[9] Jacoboni C and Lugli P 1989 The Monte Carlo Method for Semiconductor Device Simulation (New York: Springer)

[10] Barthel J M, Krienke H and Kunz W 1998 Physical Chemistry of Electrolyte Solutions (New York: Springer)

[11] Berry R S, Rice S and Ross J 2000 Physical Chemistry 2nd edn (Oxford: Oxford University Press)

[12] Rowley R L 1994 Statistical Mechanics for Thermophysical Property Calculations (Englewood Cliffs, NJ: Prentice-Hall) 
[13] Nadler B, Hollerbach U and Eisenberg R S 2003 Dielectric boundary force and its crucial role in gramicidin Phys. Rev. E 68021905

[14] Corry B, Kuyucak S and Chung S 2003 Dielectric self-energy in Poisson-Boltzmann and Poisson-Nernst-Planck models of on channels Biophys. J. 84 3594-606

[15] Mamonov A, Coalson R, Nitzan A and Kurnikova M 2003 The role of the dielectric barrier in narrow biological channels: a novel composite approach to modeling single channel currents Biophys. J. 84 3646-61

[16] Gillespie D, Nonner W, Henderson D and Eisenberg R S 2002 A physical mechanism for large-ion selectivity of ion channels Phys. Chem. Chem. Phys. 4 4763-9

[17] Bockris J and Reddy A 1998 Modern Electrochemistry 2nd edn, vol 1 (New York: Plenum)

[18] Selberherr S 1984 Analysis and Simulation of Semiconductor Devices (New York: Springer)

[19] Henderson D 1992 Fundamentals of Inhomogeneous Fluids (New York: Dekker)

[20] Schuss Z 1980 Theory and Application of Stochastic Differential Equations (New York: Wiley)

[21] Schuss Z, Singer A, Nadler B and Eisenberg R S 2004 The singlet and pair correlation functions of interacting particles in non-equilibrium diffusion: equations and boundary conditions Phys. Rev. E at press

[22] Ebeling W, Feistel R, Kelbg G and Sandig R 1978 J. Non-Equilib. Thermodyn. 311

[23] Allen R, Hansen J-P and Melchionna S 2001 Electrostatic potential inside ionic solutions confined by dielectrics: a variational approach Phys. Chem. Chem. Phys. 3 4177-86

[24] Dufrêche J-F, Bernard O and Turq P 2000 Transport equations for concentrated electrolyte solutions: reference frame, mutual diffusion J. Chem. Phys. 116 2085-97

[25] Barthel J, Münsterer M and Buchner R 1995 Dielectric properties of water and aqueous electrolyte solutions Electrolyte Data Collection part 2 (DECHEMA Chemistry Data Series vol 12) ed G Kreysa (Frankfurt: DECHEMA)

[26] Schutz C and Warshel A 2001 What are the dielectric constants of proteins and how to validate electrostatic models Proteins 44 400-17 